\title{
Si la educación avanza, la vejez retrocede*
}

\author{
If education advances, old age recedes
}

Se a educação avança, a velhice retrocede

\section{Raúl Jimenez-Guillen**}

\section{RESUMEN}

Se describe la experiencia realizada por la Licenciatura en Pe-

Palabras clave: dagogía Gerontológica de la Universidad Autónoma de Tlaxcala, para el diseño de un modelo educativo para la atención a personas mayores y la creación de la Universidad para/con Personas Mayores.

envejecimiento, educación, autonomía, agencia.

Se identifican las características de los programas que las universidades mexicanas ofrecen a las personas mayores y sirven de base para el diseño e implementación del campo formativo de intervención-acción.

Se presenta la propuesta educativa que articula los modelos de envejecimiento exitoso y educación para toda la vida, articulados por las categorías de "autonomía," "capacidad de agencia" y "relación dialógica".

Se basa en la idea de que, si la educación avanza, la vejez retrocede, porque incide en un cambio que deja atrás la cultura de la vejez centrada en la pérdida, por una que tiene como eje el desarrollo de capacidades para construir proyectos de vejez que merecen ser vividos.

\section{SUMMARY}

We describe Gerontological Pedagogy Degree of the Universidad Key words: Autónoma de Tlaxcala's experience designing an educational ging, education, model for elderly care. The programs' characteristics offered by autonomy, agency. Mexican universities to the elderly are identified and serve as a

\footnotetext{
* "Quand l'éducation avance, l'âge de la vieillesse recule", Association Internationale des Universités du Troisième Age.

** Mexicano. Académico de carrera de Tiempo Completo de la Facultad de Ciencias para el Desarrollo Humano de la Universidad Autónoma de Tlaxcala. Integrante del Cuerpo Académico Ciencias del Envejecimiento. rauljimenezguillen@yahoo.com
} 
basis for developing and implementing the intervention-action training field. The educational proposal that articulates the models of successful aging and lifelong learning, framed by the categories of "autonomy," "capacity for agency," and "dialogic relationship," is presented. It is based on the idea that if education moves forward, old age moves backward. Leaving behind the culture of old age focused on loss, for one that has its axis the development of capabilities to build old age projects that deserve to be lived.

\section{RESUMO}

Descreve-se a experiência realizada pela Licenciatura em Peda-

Palavras-chave: gogia Gerontológica da Universidad Autónoma de Tlaxcala para o desenvolvimento de um modelo educativo para o atendimento de idosos e a criação da Universidade para/com Idosos.

São identificadas as características dos programas que as univerenvelhecimento, educação, autonomia, agência. sidades mexicanas oferecem aos idosos e servem de base para o desenvolvimento e a implementação do campo formativo de intervenção-ação.

Apresenta-se a proposta educativa que articula os modelos de envelhecimento com êxito e educação para a vida toda, articulados pelas categorias de "autonomia", "capacidade de agência" e "relação dialógica".

Toma-se como base a ideia de que, se a educação avança, a velhice retrocede, porque incide em uma mudança que deixa para trás a cultura da velhice centrada na perda, por uma que tem como eixo o desenvolvimento de capacidades para construir projetos de velhice que merecem ser vividos. 


\section{Introducción}

Ante la falta de una política educativa, que atienda a la población de 60 años y más en el tema de educación, es común que los gobiernos local y federal exploten cualquier resultado, por ejemplo:

"Juana Hurtado de 96 años, concluyó sus estudios de primaria y secundaria en dos semanas, alcanzando un promedio de 9.5 en los exámenes finales que presentó..."

“José Sabino Odilón Xochipiltecatl Carvente (85 años) inició la primaria y la concluyó en diciembre de 2017. Recordó que sus hijos... me ofrecieron, si terminaba este nivel, que en mi cumpleaños me contratarían un buen grupo musical, así es que yo terminé la secundaria con un promedio de $8.7^{\prime 2}$.

El sistema de educación pública del Estado Mexicano se organiza sobre la base de grupos de edad, lo que determina que la edad máxima que aceptan las instituciones educativas es de 29 años. Las personas que no concluyen la etapa de educación básica en la edad requerida se les denomina adultos a partir de los 16 años, y esto tiene como propósito su alfabetización.

Para atender esta población, el gobierno mexicano crea, en 1950, el Centro de Cooperación Regional para la Educación de Adultos en América Latina y el Caribe (CREFAL), con el objetivo de ampliar la tarea de alfabetización que, por falta de servicios de educación pública, impide a la población acceder a la lectura y la escritura.

La alfabetización en México pasa por 3 momentos, el primero se refiere a la:

...destreza elemental hacia la noción de alfabetización funcional... [el segundo se sitúa] ... como un continuum de habilidades y conocimientos, en el cual es posible y necesario distinguir niveles de logros en lectura, escritura y matemáticas... [el tercero] se ubica en la perspectiva del aprendizaje permanente, en el cual la alfabetización comienza a ser vista como la puerta de entrada

1 https://elbigdata.mx/mexico/a-los-96-anos-mujer-termina-la-secundaria-en-chihuahua

2 https://www.elsoldetlaxcala.com.mx/local/avanza-tlaxcala-contra-analfabetismo-1868337.html 
al aprendizaje a lo largo de la vida. (Oreal/Unesco, 2013, p. 14) (cursivas del autor)

Como afirma Roberto Ham (2014), la vejez y el envejecimiento humano es producto de la civilización, lo que se refleja en el incremento de la esperanza de vida al nacer y la longevidad, que hace que el grupo de edad de mayor crecimiento en el siglo XXI sea el de de 60 años y más.

Este grupo de población tiene acceso a la educación a través de la modalidad de educación permanente, reconceptualizada como "educación para toda la vida". Considerada como elemento "clave para hacer frente a la desventaja social y superarla, promoviendo no solo la capacidad de aspiración de las personas, sino, más importante aún, sus posibilidades de escapar de la marginación" (Vargas, 2017, p. 14).

Uno de los espacios que se abren a esta población son los programas universitarios para personas mayores, que se implementan en tres momentos: el primero, con la "creación de la Universidad de la Tercera Edad", el segundo con el reconocimiento de "la dimensión educativa en las personas mayores" y el tercero "con el desarrollo de los centros de Educación de Personas Adultas (EPA), las Aulas de la Tercera Edad y los Programas Universitarios para Mayores (PUM)" (Amigo, Lázaro y Moreno, 2016, pp. 24-25).

\section{Programas Universitarios para Mayores en México 2000-2020}

En México, Saldaña y Hernández realizan una revisión de los programas educativos que se ofrecen a los adultos mayores, en el que destacan que:

En 2009, se crea la primera universidad de la Tercera Edad en la Ciudad de México, en la Delegación Benito Juárez, ... En el 2010, la Unidad de Investigación en Gerontología de la Facultad de Estudios Superiores Zaragoza, inició el Curso Universitario de Envejecimiento Activo para Adultos Mayores (CUEAAM)... La Universidad Autónoma de Nuevo León (UANL), en 2011, inició el trabajo de la Universidad para los Mayores... La Universidad Binacional de la Tercera Edad (U3E), es otra universidad que se localiza en el 
Estado de Nuevo León... dirigida por la Universidad Regiomontana, A.C. (UR) [y] el Centro de Servicios Integrales para el Adulto Mayor A.C... La Universidad Iberoamericana en Puebla ofrece un programa universitario que tiene como objetivo fortalecer el conocimiento y la experiencia que los adultos mayores; a través de un Diplomado de cuatro semestres... La Universidad Marista de Mérida, ofrece un Diplomado de cuatro semestres... [y] La Universidad del Adulto Mayor de Oaxaca (UNIDAM), con el Proyecto Diplomado del Adulto Mayor Anfitrión de Oaxaca. (Saldaña y Hernández, 2017, pp. 4-6)

Para actualizar la información, en 2020 llevamos a cabo cinco acciones: (1) con las categorías de "universidad para mayores", "universidad de la tercera edad", "universidad de la vida", "universidad de la experiencia", "aula para mayores", "aulas para la tercera edad", "aulas de la experiencia", "programas educativos para adultos mayores", "programas educativos para la tercera edad" y "programas educativos para los abuelos", se realiza la búsqueda en las plataformas de Google y Facebook. (2) Con los programas localizados, visitamos su página web y/o establecemos comunicación con la institución para solicitar información y el envío del programa con los requisitos de ingreso y egreso y costos. (3) Se efectúa una segunda búsqueda, en las plataformas de publicaciones periódicas, acerca de programas educativos que atienden a personas mayores. (4) Se elabora una matriz con cinco elementos: año de creación, nombre del programa, objetivo, oferta educativa, requisitos de ingreso y egreso, y costos, y (6) se procede a sistematizar y redactar el informe.

Hasta noviembre de 2020 se localizan 18 programas - 11 más que los reportados por Saldaña y Hernández-; ocho de los programas son ofrecidos por universidades públicas: Universidad Nacional Autónoma de México (UNAM), Universidad Autónoma de Baja California (UABC), Universidad Autónoma del Estado de México (UAEMx), Universidad Autónoma de Nuevo León (UANL), Universidad de Guadalajara (UdeG), Benemérito Universidad Autónoma de Puebla (BUAP), Universidad Autónoma de Yucatan (UAdY) y Universidad Mexiquense (UMex); cuatro por universidades privadas: Universidad Regiomontana (UR), Universidad Iberoamericana-Puebla (UIA-Puebla), Universidad Marista (UM) y Universidad del Sur (UNASUR); cuatro por orga- 
nismos de la administración pública, las delegaciones - hoy alcaldías del Distrito Federal- (Xochimilco y Benito Juárez) y dos por los Sistemas Estatales para el Desarrollo Integral de la Familia (DIF Puebla y Edomex).

Siete de los programas son reconocidos como universidades, aunque solo dos tienen existencia formal como tales: Universidad del Adulto Mayor A. C. (Oaxaca) y Universidad Mexicana para Personas Mayores (INAPAM-DIF-Fundación Paula Briones). La Universidad para los Mayores (UANL), la Universidad de la Tercera edad (U3E/CESIAM/Universidad Regiomontana), la Universidad Xochimilco para Adultos Mayores (UXAM/DIF), la Universidad de los Mayores (UAdeY) y la Universidad de la Tercera Edad (actualmente Centro de Educación Continua para Adultos Mayores (DBJ/CDMx) operan como programas de las instituciones a las que pertenecen.

Con relación al año de creación, el primer programa para adultos mayores en una universidad aparece en 2002 y el último en 2018. Al organizarlos en grupos quinquenales, entre 2000 y 2005 se crea un programa, entre 2006 y 2010 nacen cinco, entre 2011 y 2015 aparecen ocho y entre 2016 y 2020 surgen cuatro programas.

Seis de los programas son ofrecidos con ese nombre: Programa Abuelos Lectores y Cuentacuentos (UNAM), Programa de Educación Sustentable para Adultos Mayores (UABC), Programa Universitario de Envejecimiento Activo (UAEMx), Programa Universitario para Adultos 50 y Más (BUAP), Programa Universitario para Adultos (UIAPuebla) y Programas para Adultos Mayores (Universidad Marista Yucatán).

Tres adoptan el nombre de una estructura organizacional y/o administrativa: Sistema Universitario del Adulto Mayor (UdeG), Unidad de Desarrollo y Educación para la Tercera Edad (DIF-Edomex) y Unidad de Estudios Superiores para Adultos Mayores (Universidad Mexiquense del Bicentenario).

Dos toman el nombre de la propuesta curricular: Curso Universitario de Envejecimiento Activo para Adultos Mayores (Facultad de Estudios Superiores Iztacala UNAM) y Diplomado Envejecimiento Exitoso (Escuela Nacional de Trabajo Social UNAM). 
Los objetivos que refieren los programas permiten ordenarlos en cuatro grupos:

1) Los que desarrollan habilidades para cumplir tareas específicas como: capacitar a los abuelos lectores como promotores de la lectura $^{3}$, "formar ciudadanos capaces de desarrollar actitudes y propuestas de solución para enfrentar los problemas que padecen la sociedad y el medio ambiente" .

2) Aquellos que proporcionan conocimientos sobre el proceso de vejez/envejecimiento: "Proporcionar al adulto mayor un programa de formación para propiciar su desarrollo integral durante la vejez en el marco del envejecimiento activo" ${ }^{5}$. "Dar oportunidad a los adultos mayores de continuar su desarrollo, conviviendo con sus iguales y decidiendo sobre su propio proceso de formación mediante un programa educativo flexible, incluyente, dinámico e integral, que contribuya a dignificar esta etapa de su vida y fortalecer su autonomía"6. "Asegurar un desarrollo integral, especializado en el ámbito gerontológico a través de materias, talleres y cursos, en el Centro de Educación Continua para Adultos Mayores (CECAM), que comprendan las necesidades de aprendizaje, desarrollo físico y mental de los Adultos Mayores inscritos en periodos establecidos"'?.

3) Los que inciden en la mejora y/o enriquecimiento de la etapa de vejez/envejecimiento: "Impulsar un aprendizaje flexible y creativo que permita a este grupo de la población adquirir nuevos conocimientos, actualizar los que ya posee e, incluso, alcanzar metas de desarrollo académico, profesional, laboral y personal" ${ }^{\text {. }}$. Enriquecer los conocimientos culturales, artísticos y de vida en el adulto mayor9. "Promover el desarrollo educativo y la preparación académica de las personas de 60 años y más" (INAPAM, 2013, p. 16). "Contribuir a que las personas adultas mayores posean un mayor bagaje

\footnotetext{
3 https://www.jornada.com.mx/2017/03/19/cultura/a02nlcul http://fch.mxl.uabc.mx/boletin-18/

https://eventosunam.wordpress.com/category/actividad/curso/page/2/

http://unidamoaxaca.blogspot.com/p/f.html

http://www.delegacionbenitojuarez.gob.mx/node/147

http://148.228.56.113/cincuentaymas/50ymas_files/7kl217yz_files/bienvenida.html https://www.marista.edu.mx/educacion-continua/curso/97/diplomado-formacion-humana-integral-adultos-mayores/key:cursos
} 
de conocimientos que les permita comprender los cambios individuales que están viviendo y los aspectos que en el entorno se generan por el envejecimiento de la población" (Casas, 2011, p. 550), o "mejorar su calidad de vida y prepararlos para vivir un proceso de envejecimiento exitoso" ${ }^{\prime 1}$.

4) Aquellos que se constituyen en espacios de intercambio: "Proporcionar un espacio de formación oportuna y flexible, sin exigencia de grado escolar o laboral" ${ }^{11}$. Construir un espacio de educación superior para adultos y adultos mayores, y "Generar espacios de vinculación participativa universidad y comunidad que contribuyan a la reflexión y la valorización de las capacidades propias del adulto mayor" (Ciano, 2017, p. 80).

La oferta educativa de todos los programas puede agruparse en cuatro modalidades: conferencias, cursos, talleres y diplomados.

En las conferencias se toma como referencia el programa de $50 \mathrm{y}$ más de la $\mathrm{BUAP}^{12}$, que en otoño de 2018 ofrece: conferencias, psicología positiva, programa neurolingüística para vivir excelentemente, adultos con actitud positiva, el sentido de nuestras decisiones y el arte de vivir, taichí, promoción y prevención de enfermedades crónico degenerativas, nutricio 50 y más, cine e historia de México, taller de celular inteligente nivel básico, persona y sentido de la vida, cosmovisión, ciencia y religión en el mundo prehispánico, el cerebro digestivo después de los 50, el don de ser abuelo, aprendizaje del idioma inglés para la vida diaria y taller de celular inteligente.

La oferta del Centro de Educación Continua para Adultos Mayores $^{13}$ (CECAM), inicialmente creada como Universidad de la Tercera Edad, permite mostrar la variedad de cursos: manejo de nuevas tecnologías, comunicación mediada por tecnología, ambientes virtuales de aprendizaje, comunidades y redes sociales, derecho, cultura en la edad media, bailes finos de salón, danzón, baile tropical, herbolaria mexicana y temazcalli, inteligencia emocional, logo-

10 https://www.elfaromx.com/post/2017/06/16/porque-nunca-es-tarde-para-aprender-adultos-mayores-se-grad\%C3\%BAan-en-nl

11 http://www.udg.mx/es/secciones/informacion-general?page=33

12 http://www.50ymas.buap.mx/

13 http://www.delegacionbenitojuarez.gob.mx/node/147 
terapia aplicada, activa tu mente con calidad de vida, vitromosaico, apreciación cinematográfica, autoconocimiento en la tercera edad, autoestima y motivación, búsqueda de la felicidad, crónicas de la ciudad de México, pueblos mágicos, dígito presión, el arte de narrar, historia comparada de las religiones, el hombre a través de sus mitos leyendas y tradiciones, flores de Bach, historia universal, historia de México, historia del arte, inteligencia emocional, literatura, proceso del perdón, reflexología, resiliencia, equilibrio y seguridad en la vejez y taller de activación de la memoria.

Talleres (se toma como referencia el programa universitario de envejecimiento activo del Centro Universitario Ecatepec, UAEMx): "uso y manejo de las nuevas tecnologías (computadora, internet y celular), actividades artísticas (psicodanza, canto, cine) y deportivas (activación física, caminatas, yoga), actividades lúdicas (juegos tradicionales mexicanos, juegos virtuales), académicas (conferencias, debates, investigación), de aprendizaje en comunidad (visitas a domicilio, para conocer y aprender de los saberes de las personas de edad) y de fomento de la salud (análisis de los factores asociados a la vida saludable y práctica de la vida saludable)" (Aldana et al., 2016, p. 59).

Diplomados: desarrollo sustentable (UABC), envejecimiento exitoso (ENTS-UNAM), desarrollo personal y humano (Universidad Regiomontana), desarrollo especializante del adulto mayor (UdeG) y formación humana (Universidad Marista).

Entre los requisitos de ingreso, el de mayor peso es la edad, con excepción de una institución que exige 35 años, el resto (17 programas) establecen como mínimo 50 (6), 55 (5) o 60 (6) años de edad. El segundo requisito es saber leer y escribir; aunque algunos consideran la educación básica, generalmente solo se requiere para los diplomados. El tercero, valerse por sí mismo, lo que determina que solamente se atiende a personas mayores funcionales, y el cuarto, cubrir las cuotas de inscripción y colegiatura.

En lo que se refiere al egreso, todos los programas, sin excepción, otorgan diplomas sin validez curricular, pues, como señalan los directivos de la UABC, "no podemos darle un título de..."

En los costos se registran diferencias entre las universidades públicas y privadas, ya que algunas de las primeras ofrecen servicios gratui- 
tos (UdeG) o con costos simbólicos de 100 a 300 pesos, en tanto que en las privadas oscilan entre 2 mil y 18 mil pesos. El costo promedio de los diplomados en las universidades públicas es de 2/3.5 mil pesos. Las universidades privadas establecen costo por unidad de aprendizaje y estos van de 3 a 16 mil pesos.

Algunos programas que no tienen costo establecen como criterio la reciprocidad, esto es, que las personas mayores deben realizar servicio social a favor de otros grupos de personas, por ejemplo, el de cuentacuentos o trabajar con niños.

Derivado de lo anterior, pueden reconocerse cinco características comunes de los programas universitarios y/o universidades para personas mayores:

1. Son programas diseñados, promovidos y coordinados por instancias administrativas universitarias, particularmente en las áreas de extensión y/o educación continua.

2. Se dirigen a personas mayores con tres edades de referencia: 50, 55 y 60 años.

3. La oferta se integra por cursos, talleres y diplomados, orientados al desarrollo personal, ocio cultural e integración social.

4. Los requisitos de ingreso son: saber leer y escribir, estar sano y pagar los costos de inscripción y colegiaturas. Solo algunos diplomados exigen el certificado de educación media superior.

5. Al término de los estudios se otorgan diplomas o constancias de participación o reconocimiento, sin valor curricular.

Los programas educativos que las universidades ofrecen a las personas mayores no están orientados hacia la alfabetización, sino a la ocupación del tiempo libre. Están dirigidos a la población de clase media en el marco de la educación para toda la vida. Tomando como base estas referencias, en el Estado más pequeño de la República mexicana, la universidad estatal diseña e implementa un programa para orientar la intervención del proceso formativo para profesionales en pedagogía gerontológica. 


\section{La intervención-acción para y con personas mayores}

En 2012, la Facultad de Ciencias para el Desarrollo Humano de la Universidad Autónoma de Tlaxcala crea la licenciatura en Atención Integral al Adulto Mayor, basada en los principios y metodologías de la pedagogía gerontológica.

El programa educativo se propone formar profesionales capaces de diseñar, gestionar, evaluar y promocionar metodologías socioeducativas de intervención, para mejorar la calidad de vida de las personas mayores. El campo formativo de intervención-acción profesional:

implica el accionar directo del profesional en la puesta en marcha y ejecución de las propuestas de intervención diseñadas desde los campos formativos de Política y Gestión y Métodos de Intervención Socioeducativa. El trabajo directo con los adultos mayores deberá de ser justificado y presentado desde el análisis de la realidad, la reflexión, la ejecución e interpretación de la práctica y la evaluación inicial, procesual y final de la acción integrándolas en un informe final de la experiencia. (LAIAM, Plan 2012, p. 19)

Como parte de las actividades de este campo formativo, entre 2015 y 2018 se desarrollan seis experiencias — tres ciclos de conferencias, dos talleres y un seminario - para acercar a los estudiantes con las personas mayores. Las temáticas de los ciclos de conferencias son:

1) Políticas públicas para la atención de las personas mayores. Se invita a las delegaciones de siete organismos federales (SESA, IMSS, ISSSTE, SEDESOL, INEA, ICATLAX y CEDH) para presentar los programas y servicios que ofrecen, con el propósito de conocer la forma en que el Estado mexicano implementa la política pública para la atención hacia las personas mayores.

2) Vejez, Religión y Muerte. El propósito de este segundo ciclo es desplegar los distintos marcos de referencia religiosa con los que se explica la muerte. Para ello se invita a siete sacerdotes, rabinos y/o pastores (iglesia católica, testigos de Jehová, mormones, pentecostales y espiritualistas).

3) La experiencia de personas mayores exitosas. Basados en el modelo de inteligencias múltiples de Gardner (2005), se convida a siete personas (cinco mujeres y dos hombres) que, entre sus pares, son 
considerados como exitosas en las áreas de las bellas artes, humanidades y ciencias, para compartir su experiencia en el proceso de convertirse en profesionales destacados.

En 2016 se implementa el primer taller para personas mayores, al que asisten 20 mayores de 60 años. La intención es ofrecer, a través de la lectura, posibilidades de construcción de reservas cognitivas y, a la vez, implementar el primer proyecto de investigación-acción, con el propósito de medir los cambios que se producen en la capacidad cognitiva y la depresión. Como producto se elabora una tesis de grado que obtiene mención honorífica.

En 2017 se realiza un segundo taller, con el propósito de que los estudiantes interactúen con personas mayores compartiendo historias de vida, y realicen una actividad práctica diseñada para ellas por los estudiantes. Asisten 20 adultos mayores y 50 estudiantes. Se realiza una tesis de grado que obtiene mención honorífica.

En 2018, aprovechando la experiencia y participación de las personas mayores, se incorpora una de ellas como académica honoraria y se le pide que, dentro de su círculo de amistades, invite a participar a 15 personas para compartir sus historias de vida. La idea es que los estudiantes escuchen a un grupo social diferente de aquel con que interactúan en las prácticas escolares y el servicio social, ya que se trata de personas de nivel socioeconómico C+ Medio Alto, de acuerdo con la clasificación INEGI.

Basados en los resultados, se plantea la ampliación de la experiencia a partir de tres ideas: 1) la concepción de envejecimiento exitoso (Rowe y Kahn, 1987, 1997) en sus tres dimensiones; 2) la incorporación de personas mayores como facilitadores (maestros honorarios del programa) y 3) la prestación de servicios a las personas mayores como parte de un proceso educativo.

Se busca crear un espacio de encuentro para promover la formación de redes sociales, compartir entre pares experiencias vitales, estimular la memoria a partir de experiencias personales, fortalecer la capacidades cognitivas y funcionales, e intensificar el compromiso con la vida.

Se diseñan cinco talleres, cada uno con un profesor honorario una persona mayor que participa de los dos primeros talleres-, con la 
idea de que esta experiencia se convierta en parte del componente de compromiso con la vida, y se incorporan estudiantes de la Licenciatura en Atención Integral del Adulto Mayor para la realización de prácticas y servicio social (ver cuadro 1).

Cuadro 1.

Propuesta integrada de la primera experiencia para crear la Universidad para/con Personas Mayores.

\begin{tabular}{|c|c|c|c|c|c|}
\hline \multirow[t]{4}{*}{$\begin{array}{l}\text { Envejecimiento } \\
\text { exitoso }\end{array}$} & \multicolumn{2}{|c|}{$\begin{array}{l}\text { Baja probabilidad de } \\
\text { enfermedad y discapacidad } \\
\text { relacionada con la } \\
\text { enfermedad }\end{array}$} & \multicolumn{2}{|c|}{$\begin{array}{l}\text { Alta capacidad funcional } \\
\text { cognitiva y física }\end{array}$} & \multirow{3}{*}{$\begin{array}{l}\text { Compromiso } \\
\text { con la vida } \\
\text { Talleres } \\
\begin{array}{l}\text { Con dinero y } \\
\text { sin dinero }\end{array}\end{array}$} \\
\hline & Talleres & & Talleres & & \\
\hline & $\begin{array}{l}\text { Sentir el } \\
\text { cuerpo }\end{array}$ & $\begin{array}{l}\text { La carpa de } \\
\text { los recuerdos }\end{array}$ & $\begin{array}{l}\text { Lecturas } \\
\text { contra el } \\
\text { olvido }\end{array}$ & $\begin{array}{l}\text { Donde se } \\
\text { cocina la } \\
\text { vida }\end{array}$ & \\
\hline & Meditación & Títeres & Lectura & Cocina & $\begin{array}{l}\text { Finanzas } \\
\text { personales }\end{array}$ \\
\hline Objetivo & $\begin{array}{l}\text { Disminuir } \\
\text { los niveles de } \\
\text { estrés para } \\
\text { alejarse de la } \\
\text { enfermedad y } \\
\text { discapacidad } \\
\text { relacionada } \\
\text { con la } \\
\text { enfermedad }\end{array}$ & $\begin{array}{l}\text { Robustecer } \\
\text { la capacidad } \\
\text { funcional } \\
\text { física a } \\
\text { través de la } \\
\text { coordinación } \\
\text { motora fina }\end{array}$ & $\begin{array}{l}\text { Explorar el } \\
\text { proceso de } \\
\text { investigación } \\
\text { para } \\
\text { fortalecer la } \\
\text { capacidad } \\
\text { funcional }\end{array}$ & $\begin{array}{l}\text { Degustar } \\
\text { alimentos } \\
\text { como } \\
\text { medio para } \\
\text { reanimar la } \\
\text { capacidad } \\
\text { funcional } \\
\text { cognitiva }\end{array}$ & $\begin{array}{l}\text { Revisar las } \\
\text { finanzas } \\
\text { personales } \\
\text { para construir } \\
\text { proyectos } \\
\text { de vida } \\
\text { productiva }\end{array}$ \\
\hline Profesor honorario & $\begin{array}{l}\text { Ofelia } \\
\text { Hernández } \\
\text { Rodríguez }\end{array}$ & $\begin{array}{l}\text { Enedina } \\
\text { Barbosa } \\
\text { Minor }\end{array}$ & $\begin{array}{l}\text { Guillermo } \\
\text { Pérez Cruz }\end{array}$ & $\begin{array}{l}\text { Esperanza } \\
\text { Portano } \\
\text { Mondragón }\end{array}$ & $\begin{array}{l}\text { José Santos } \\
\text { Rivera }\end{array}$ \\
\hline $\begin{array}{l}\text { Estudiantes de la } \\
\text { Licenciatura en } \\
\text { Atención Integral } \\
\text { del Adulto Mayor }\end{array}$ & $\begin{array}{l}\text { Cristal Pérez } \\
\text { Cuellar, } \\
\text { Susana } \\
\text { Corona } \\
\text { Ordaz }\end{array}$ & $\begin{array}{l}\text { Yamileth Fabi } \\
\text { Nava Sastré, } \\
\text { María José } \\
\text { Flores } \\
\text { Gutiérrez }\end{array}$ & $\begin{array}{l}\text { Luis Mario } \\
\text { Fernández } \\
\text { Loyo, } \\
\text { Diana García } \\
\text { Pérez }\end{array}$ & $\begin{array}{l}\text { Andrea } \\
\text { Hernández } \\
\text { Arenillas, } \\
\text { Abril Pluma } \\
\text { Nava. }\end{array}$ & $\begin{array}{l}\text { María } \\
\text { Cándida } \\
\text { Ramírez Cruz }\end{array}$ \\
\hline
\end{tabular}

Fuente: elaboración propia.

\section{Propuesta educativa para crear la Universidad para/ con Personas Mayores}

Derivado de esta experiencia, al integrar el trabajo con personas mayores en una serie de talleres, se considera pertinente la creación de un espacio propio del programa que cumpla tres propósitos: brindar de manera permanente servicios educativos a las personas mayores, contar con un espacio propio para las prácticas profesionales y el servicio social, y crear experiencias innovadoras de intervención que promuevan la investigación. 
El eje central del modelo es lo pedagógico, porque se busca cambiar la concepción de que la educación es el proceso de culturización de una generación madura a otra que no lo está. Esta visión vertical se horizontaliza en razón de que las personas mayores han vivido 60 años o más, y tienen conocimientos y experiencias que pueden compartir, pero también aprender a construir su propio envejecimiento de manera diferente.

Los que distingue esta propuesta de las que ofrecen otras universidades públicas del país está determinado por la posición del sujeto en el proceso educativo. Se trata de crear comunidades intergeneracionales que modifiquen la visión individual y social de la vejez y el envejecimiento patológico, y muestren, en la práctica, la posibilidad de construir otras vejeces.

La propuesta se diseña a partir de dos modelos: 1) envejecimiento exitoso y 2) educación para toda la vida, articulados a través de las categorías de "autonomía", "capacidad de agencia" y "relación dialógica" (ver imagen 1).

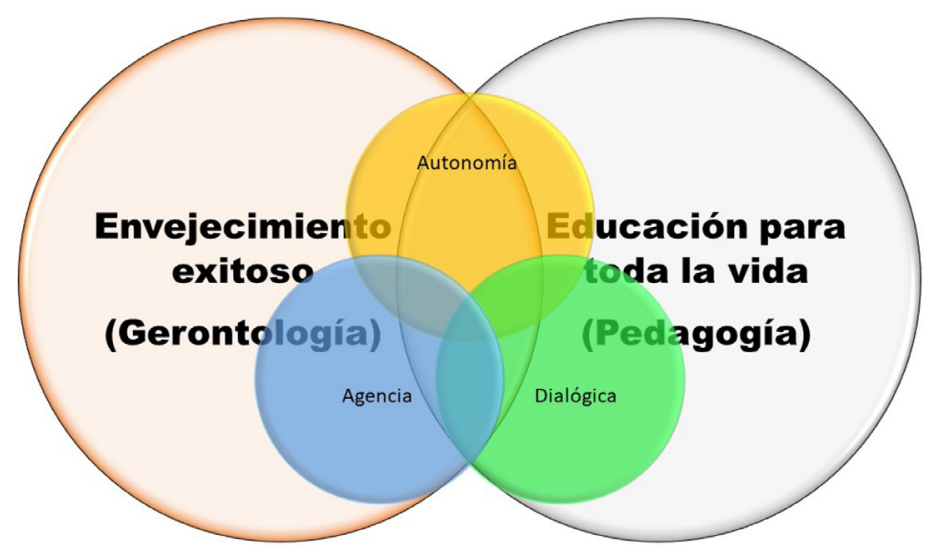

Modelo educativo de la universidad para/con Personas Mayores de la FCDHUATx, RJG, 2020.

\section{Envejecimiento exitoso}

Rowe y Kahn (1987 y 1997) refieren que geriatras y gerontólogos tienden a interpretar los déficits cognitivos y fisiológicos asociados con la edad como determinados por la misma. Lo que ha contribuido en la 
construcción de una cultura de la vejez asociada con la pérdida, el déficit, el deterioro, la discapacidad y la dependencia.

En la década de los 80 del siglo $\mathrm{XX}$, la red de investigación de la Fundación MacArthur permitió observar tres tipos de envejecimiento: patológico, normal y exitoso. El primero responde al tipo de personas con enfermedades y discapacidades que han perdido su autonomía; la segunda, a personas que registran enfermedades y desgastes fisiológicos y funcionales normales de la edad, pero que mantienen una vida independiente, y la tercera a quienes no presentan o presentan muy pocas enfermedades o disfunciones, y mantienen un alto compromiso con la vida.

El concepto de "envejecimiento exitoso" tiene su origen en la teoría de la actividad de la década de los 50 del siglo XX, pero es a partir de la propuesta de Rowe y Kahn que se utiliza ampliamente - junto con otros muy relacionados con esta visión optimista del envejecimientocomo "envejecimiento activo", "envejecimiento positivo", envejecimiento saludable" y "envejecimiento óptimo" (Cosco, Prina, Stephan \& Brayne, 2013; Cheng, 2014; Cosco, 2015).

El envejecimiento exitoso de define a partir de tres componentes: baja probabilidad de enfermedad y discapacidad relacionada con la enfermedad, alta capacidad funcional cognitiva y física, y compromiso activo con la vida.

\section{Educación para toda la vida}

El marco legal de la educación pública en México no incluye la educación para toda la vida, aunque se hace referencia a que: "En educación para personas adultas, se aplicarán estrategias que aseguren su derecho a ingresar a las instituciones educativas en sus distintos tipos y modalidades" (Art. $3^{\circ}$ Constitucional).

La categoría de "adulto" se reduce a "población de quince años o más que no hayan cursado o concluido la educación primaria y secundaria" (Art. 70 ${ }^{\circ}$. Ley General de Educación) y aunque refiere que será considerada una educación a lo largo de la vida, esto se reduce a servicios de alfabetización, educación primaria, secundaria y formación para el trabajo. 
Por lo que se toma el concepto desarrollado por la UNESCO ${ }^{14}$ :

El aprendizaje a lo largo de la vida es un principio organizativo de todas las formas de educación (formal, no formal e informal) con componentes mejor integrados e interrelacionados. Se basa en la premisa de que el aprendizaje no está confinado a un periodo específico de la vida, sino que va "de la cuna a la tumba" (sentido horizontal), considera todos los contextos en los que conviven las personas como familia, comunidad, trabajo, estudio, ocio (sentido vertical), y supone valores humanísticos y democráticos como la emancipación y la inclusión (sentido profundo). Enfatiza el aseguramiento de los aprendizajes relevantes (y no sólo la educación) más allá del sistema escolar. (Unesco, 2015, p. 2)

Ya que este tipo de educación debe "apoyar y aumentar la dignidad, la capacidad y el bienestar de la persona humana en relación con los demás y la naturaleza" (p. 35), tiene como finalidad: "el respeto a la vida y a la dignidad humana, la igualdad de derechos y la justicia social, la diversidad cultural y social, y el sentido sentimiento de la solidaridad humana y la responsabilidad compartida de nuestro futuro sostenible" (p. 9).

Como destaca Maturana, educar se constituye en el proceso por el que las personas conviven con los otros y, al convivir con los otros, "se transforman espontáneamente, de manera que su modo de vivir se hace progresivamente más congruente con el otro en el espacio de convivencia" (1988, p. 14).

\section{Autonomía}

Elemento fundamental para el trabajo educativo con las personas mayores es el respeto y fomento a la autonomía, como capacidad percibida para controlar, afrontar y tomar decisiones personales acerca de cómo vivir de acuerdo con las normas y preferencias propias, porque ello permite la participación en la construcción de un proyecto de vida que merezca ser vivido.

14 https://uil.unesco.org/fileadmin/multimedia/uil/confintea/pdf/Format_of_the _ structure_of_the_glossary.pdf 
El modelo de intervención con personas mayores se funda en los derechos humanos, a través de lo señalado por la Convención Interamericana sobre Protección de Derechos Humanos de las Personas Mayores, en la que se reconoce que "la persona mayor tiene los mismos derechos humanos y libertades fundamentales que otras personas, y que estos derechos, incluido el de no verse sometida a discriminación fundada en la edad..." ${ }^{15}$.

En el artículo séptimo de la convención se reconoce: "el derecho de la persona mayor a tomar decisiones, a la definición de su plan de vida, a desarrollar una vida autónoma e independiente, conforme a sus tradiciones y creencias, en igualdad de condiciones y a disponer de mecanismos para poder ejercer sus derechos".

Por lo que se responsabiliza al Estado, la familia y la comunidad de la "integración activa, plena y productiva de la persona mayor dentro de la sociedad, así como en su cuidado y atención, de acuerdo con su legislación interna" (Art. $3^{\circ}$ Fracción o), propuestas que recogen e integran las leyes nacional y estatales de los Derechos de la Personas Adultas Mayores, y en las que se reconoce que las acciones que realice el Estado - federación, Estado y municipios- deberán orientarse a fortalecer su independencia, su capacidad de decisión y su desarrollo personal y comunitario.

\section{Capacidad de agencia}

Amartya Sen define el desarrollo como "un proceso de expansión de las libertades reales que disfruta la gente", porque asume que "con oportunidades sociales adecuadas, los individuos pueden formar efectivamente su propio destino y ayudarse unos a otros; no necesitan ser considerados como los receptores pasivos de los beneficios de ingeniosos programas de desarrollo" (Sen, 2000, p. 28).

Las personas mayores no tienen por qué verse siempre, o casi siempre, como receptores pasivos de la acción de los demás, sean estos familiares, profesionales o programas gubernamentales. Se les debe

15 http://www.oas.org/es/sla/ddi/docs/tratados_multilaterales_interamericanos_A-70_ derechos_humanos_personas_mayores.pdf 
involucrar activamente para construir un proyecto de envejecimiento diferente (Nussbaum, 2018).

El número de años que vive una persona después de la edad considerada como de salida del mercado productivo - 65 años - es de entre cinco y 15 años, tiempo para construir un proyecto de envejecimiento que merezca ser vivido.

La agencia tiene como características que, según Alkire (2008), a) se ejerce con respecto a las metas que la persona valora; b) incluye el poder efectivo, así como el control directo; c) puede avanzar en el bienestar o puede abordar objetivos que se refieren a otros; d) implica una evaluación del valor de los objetivos del agente; y e) la responsabilidad del agente por un estado de cosas debe incorporarse en su evaluación de la misma.

Una característica particular de la vejez es que las personas mayores descubren que se tienen a sí mismas y es la ocupación - y no la preocupación- la que debe determinar cómo desean vivir esos años. Pero ello demanda de la definición de un proyecto de vida que se quiera y merezca ser vivido.

\section{Relación dialógica}

Enseñar, dice Freire (2012, p. 22), "no es transferir conocimiento, sino crear las posibilidades para su propia producción o construcción", en razón de que el ser humano se experimenta a sí mismo como un "ser cultural, histórico, inacabado y consciente del inacabamiento", y es esa incompletud la que desencadena "un proceso social de búsqueda".

De ahí que la práctica pedagógica se funda en los principios del reconocimiento de la experiencia, el diálogo y una relación horizontal, en razón de que las personas mayores que participan de esta acción llegan con un bagaje de cuando menos 60 años de experiencia de vida, lo que implica contar con una multiplicidad de saberes que se convierten en mediadores del proceso de aprendizaje.

Como refiere Freire, de ninguna manera se puede

dejar de considerar su saber hecho a partir de la experiencia. Su explicación del mundo, de la que forma parte la comprensión de su propia presencia en el mundo. Y todo eso viene explícito o sugerido 
o escondido en lo que llamo lectura del mundo, que procede siempre a la lectura de la palabra. (p. 37)

El supuesto de que la educación es la transmisión de la cultura de una generación madura a una que no lo está, modifica la relación entre los actores del proceso educativo. En este caso, las generaciones se ubican en un plano de horizontalidad, y cada uno aporta para la construcción de un proyecto de envejecimiento activo, saludable.

La relación pedagógica está determinada por el diálogo, como encuentro de seres humanos que pronuncian el mundo, en un espacio en el que confluyen informaciones y conocimiento diferentes, pero que se convierten en los mediadores para construir un proyecto esperanzador en una vejez diferente que modifique la visión de sí mismos y contribuyan al cambio de una cultura del envejecimiento.

La Universidad para/con Personas Mayores, en consonancia con la misión de la universidad de hacer que por la cultura se alcance la justicia social, establece como propósito el desarrollo de capacidades de las personas mayores para que, a través de la educación, la vejez patológica retroceda.

\section{Conclusiones}

En México, la educación para las personas mayores aparece con los albores del siglo XXI, momento en que el Congreso de la Unión aprueba la primera ley de derechos de las personas adultas mayores (2002) y transforma la institución encargada de estas tareas, que pasa de Instituto Nacional de la Senectud, Instituto Nacional de Adultos en Plenitud, a Instituto Nacional para la Atención de Personas Adultas Mayores. Aunque es una ley "incompleta", ya que no cuenta con instancias y procesos a lo que los que las personas mayores puedan acudir para hacer efectivos los derechos contenidos en las leyes.

La educación que se ofrece a las personas mayores deviene de la experiencia de la alfabetización funcional que el gobierno federal impulsa, a efectos de paliar la falta de escuelas en las décadas del 60 y 70 del siglo XX. Hacia la década de los 90 se transforma en la atención del rezago, considerando adultos mayores a las personas de 16 años que no concluyen la educación básica y, para ello, crea una institución paralela a la Secretaría de Educación Pública - el Instituto Nacional de 
Educación para los Adultos, INEA-, que marginalmente atiende a la población mayor de 60 años.

Los casos de atención que registra el INEA se convierten en referentes públicos y son publicitados a efectos de mostrar que las personas mayores también son atendidas, pero no es sino hasta 2018 que propone trabajar "la vertiente para la atención de las personas adultas mayores, dirigida a la población de 60 años y más, a fin de que dichas personas aprendan a leer y escribir o concluyan su educación primaria o secundaria"16.

Los programas de atención a personas mayores en las universidades inician en 2002, con cercanía al modelo francés.

El modelo francés se basa en la creación de Universidades de la Tercera Edad dentro de las universidades, principalmente universidades públicas, como departamento o facultad. Este modelo también incluye universidades de la Tercera Edad directamente afiliadas a universidades, principalmente universidades públicas. (Vellas, $\mathrm{s} / \mathrm{f})^{17}$

De los 18 programas localizados, 14 son parte de la función de extensión universitaria educación continua o responsabilidad social, que las universidades públicas tienen para con la sociedad y los implementan como programas de "educación continua o extensión universitaria". Este tipo de "servicio educativo está pensado en la perspectiva del ocio cultural, que trata de ocupar a los mayores y favorecer su integración social" (Lara, 2014, p. 115).

Todos los programas universitarios están diseñados en la lógica de que la institución es la que ofrece y enseña contenidos a los adultos mayores. "La experiencia de asistir a la Universidad como aprendiz" (Aldana et al., 2016, p. 60).

La diferencia fundamental es que, al no exigir como requisito de ingreso más que saber leer y escribir, determina que no se otorgue reconocimiento formal a los estudios realizados, ya que se parte de la idea de que los adultos mayores no acuden a la universidad a "obtener grados

16 Comunicado de prensa de 17 de abril de 2018.

17 International Association of Universities of Third Age. Comparison between the French Model and the British Model. Recuperado de https://www.aiu3a.org/pdf/Paper7.pdf 
o títulos", sino "sabiduría". Lo que a su vez determina que la mayor parte de los programas - de acuerdo con sus objetivos- están diseñados para establecer, o reproducir, una relación vertical entre quienes son considerados como los poseedores del conocimiento (las instituciones y los profesores) y los que no cuentan con conocimiento y acuden a "aprender" (las personas mayores). En ningún momento los programas reconocen la experiencia - aunque algunos de ellos adopten ese título- ni el conocimiento y, por tanto, los adultos mayores son visualizados, incorporados y tratados como "alumnos". Los títulos de las conferencias, cursos, talleres - y aun de los diplomados- proporcionan conocimientos generales, más como una ocupación del tiempo libre o de ocio, que con una idea de educación permanente.

En ninguna de las universidades se incorporan adultos mayores como los diseñadores, implementadores, coordinadores o monitores de los programas. La mayor parte de los docentes son jóvenes, no hay adultos mayores, como si no existieran experiencias que compartir o como si la vida se les hubiera pasado en el vacío.

Esto es lo que determina realizar un proceso de búsqueda en la Licenciatura de Atención Integral del Adulto Mayor - hoy Pedagogía Gerontológica - para crear un modelo educativo que permita que, a través de la Universidad para/con Personas Mayores, participen en la construcción de un proyecto de envejecimiento activo y saludable.

\section{Bibliografía}

Amigo, F. M., Lázaro, F, y Moreno N. I. (2016) Principios educativo ocio humanista en los programas de formación permanente de adultos de las universidades españolas. Inguruak, (61), 2342.

Aldana G. et al. (2016). Construcción social del sentido de comunidad en integrantes de un programa universitario de envejecimiento activo. Diotima, 1(2), 56-71.

Alkire, S. (2008). Concepts and measures of agency. OPHI Working Paper 9, University of Oxford.

Casas, T. G. (2011). Diplomado envejecimiento exitoso. IV Congreso Iberoamericana de Universidades para Mayores, Tomo I, Alicante, 547-555. 
Cheng, S. (2014). Defining successful aging: the need to distinguish pathways from outcomes. International Psychogeriatrics, 26(4), 527-531.

Ciano, N. (2017). Proceso específico de orientación para adultos mayores (Tesis Doctoral). Universidad Nacional de la Plata, Argentina.

Cosco, T., Prina M., Stephan, B. \& Brayne, C. (2013) Operational definitions of successful aging: A systematic review. International Psychogeriatrics, 26(3), 1-9.

Cosco, D. (2015) Successfully aging predicts successful aging in successful Agers: Further definitional issues. International Psychogeriatrics, 27(1), 170-171.

Gardner, H. (2005) Inteligencias múltiples: la teoría en la práctica. Argentina: Paidós.

Freire, P. (2012). Pedagogía de la autonomía. México: Siglo XXI.

Ham, R. (2014). El envejecimiento en México: El siguiente reto de la transición demográfica. México: Colef/Porrúa.

Lara, L. F. (2014). Los programas universitarios para mayores: necesidad de su reconocimiento y consideración como parte integrante del Espacio Europeo de Educación Superior. (Tesis Doctoral). Universidad Autónoma de Madrid.

Maturana, H. (1988). Emociones y lenguaje en educación y política. Material para el curso de Teoría de Sistemas. Chile: Hachete/ Comunicación.

Nussbaum, M. (2018). Envejecer con sentido. Conversaciones sobre el amor, las arrugas y otros pesares. México: Paidós.

Oreal/Unesco. (2013). Lecciones desde la práctica innovadora en América Latina y el Caribe. Santiago de Chile: Unesco.

Otano, G. (2015). La libertad como relación social: Una interpretación sociológica del enfoque de las capacidades de Amartya Sen. Revista Iberoamericana de Estudios de Desarrollo, 4(1), 98127.

Rowe, J. \& Kahn, R. (1987). Human Aging: Usual and Successful. Science, 237(4811): 143-149.

Rowe, J. \& Kahn, R. (1997). Successful Aging. The Gerontologist, 37(4): 433-440.

Saldaña I., S. A. y Hernández, G. E. (2017). Universidad del adulto mayor: Un nuevo escenario de desafíos y oportunidades. XIV 
Congreso Nacional de Investigación Educativa COMIE. Recuperado de https://www.comie.org.mx/congreso/memoriaelectronica/v14/doc/2599.pdf.

Sen, A. (2000). Desarrollo y Libertad. Argentina: Planeta.

Unesco. (2015). Replantear la educación: ¿Hacia un bien común mundial? Francia: Unesco.

Vargas, C. (2017). El aprendizaje a lo largo de toda la vida desde una perspectiva de justicia social. París: Unesco.

Vellas, F. (s/f). Comparison between the french model and british model. Université du Troisiéme Age Toulouse. Recuperado de https://www.aiu3a.org/pdf/Paper7.pdf. Consultado el 21 de febrero de 2021

\section{Documentos}

Convención Interamericana sobre la protección de los derechos humanos de las personas mayores. Recuperado de http:// www.oas.org/es/sla/ddi/docs/tratados_multilaterales_ interamericanos_A-70_derechos_humanos_personas_mayores.pdf.

Censo 2020, Tlaxcala. Recuperado de https://www.inegi.org.mx/programas/ccpv/2020/\#Microdatos.

Constitución Política de los Estados Unidos Mexicanos.

INAPAM. (2013). Informe de Gestión. Primer trimestre. Recuperado de http://www.inapam.gob.mx/work/models/INAPAM/Resource/1147/1/images/InformedelDesempenoGeneraldelaEntidadPrimerTrimestre2013.pdf.

Secretaría del Trabajo y Previsión Social. Información laboral, febrero 2021. Recuperado de http://siel.stps.gob.mx:304/perfiles/ perfiles_detallado/perfil_tlaxcala.pdf.

Ley General de Educación.

Ley de los derechos de las Personas Adultas Mayores.

Ley de atención a las personas mayores del Estado de Tlaxcala.

Universidad Autónoma de Tlaxcala, Facultad de Ciencias para el Desarrollo Humano, Licenciatura en Atención Integral al Adulto Mayor. Plan 2012. Tlaxcala, México. 